\title{
A FINITE DIFFERENCE METHOD ON LAYER-ADAPTED MESHES FOR AN ELLIPTIC REACTION-DIFFUSION SYSTEM IN TWO DIMENSIONS
}

\author{
R. BRUCE KELLOGG, TORSTEN LINSS, AND MARTIN STYNES
}

\begin{abstract}
An elliptic system of $M(\geq 2)$ singularly perturbed linear reactiondiffusion equations, coupled through their zero-order terms, is considered on the unit square. This system does not in general satisfy a maximum principle. It is solved numerically using a standard difference scheme on tensor-product Bakhvalov and Shishkin meshes. An error analysis for these numerical methods shows that one obtains nodal $O\left(N^{-2}\right)$ convergence on the Bakhvalov mesh and $O\left(N^{-2} \ln ^{2} N\right)$ convergence on the Shishkin mesh, where $N$ mesh intervals are used in each coordinate direction and the convergence is uniform in the singular perturbation parameter. The analysis is much simpler than previous analyses of similar problems, even in the case of a single reaction-diffusion equation, as it does not require the construction of an elaborate decomposition of the solution. Numerical results are presented to confirm our theoretical error estimates.
\end{abstract}

\section{INTRODUCTION}

Let $M \geq 2$ be an integer. We seek the solution $\boldsymbol{u}=\left(u_{1}, u_{2}, \ldots, u_{M}\right)^{T}$ of the singularly perturbed system of $M$ reaction-diffusion equations

$$
\mathcal{L} \boldsymbol{u}:=-\varepsilon^{2} \Delta \boldsymbol{u}+A \boldsymbol{u}=\boldsymbol{f} \quad \text { in } \Omega=(0,1)^{2},\left.\quad \boldsymbol{u}\right|_{\partial \Omega}=\boldsymbol{g},
$$

where the parameter $\varepsilon$ satisfies $0<\varepsilon \ll 1$. Systems of this type are relevant, for example, to the investigation of diffusion processes in electro-analytical chemistry in the presence of chemical reactions. Under certain hypotheses on the data $A, \boldsymbol{f}$ and $\boldsymbol{g}$ we shall examine a simple finite difference scheme that generates an approximate solution of (1.1) on various layer-adapted meshes.

A problem resembling (1.1) is analysed in [4, but the hypotheses there ensure that $A$ is diagonally dominant and inverse-monotone so that the system satisfies a maximum principle, while in the current paper the only hypothesis on $A$ is Assumption 1.1 below and we make no appeal to maximum principles for systems. Thus 4 and the current paper consider distinct but overlapping classes of problems. While the numerical method of [4] is identical with that of the current paper, the

Received by the editor May 24, 2007.

2000 Mathematics Subject Classification. Primary 65N06, 65N15, 65N50; Secondary 35J45.

The research of the first author was supported by the Boole Centre for Research in Informatics at the National University of Ireland, Cork, and by the Science Foundation Ireland under the Basic Research Grant Programme 2004 (Grants 04/BR/M0055, 04/BR/M0055s1).

The research of the second author was supported by the Boole Centre for Research in Informatics at the National University of Ireland, Cork and by the ZIH at TU Dresden.

(C)2008 American Mathematical Society Reverts to public domain 28 years from publication 
analysis of 4] relies heavily on the maximum principle already mentioned, and on the construction of an elaborate decomposition of $\boldsymbol{u}$ into a sum of a smooth part, a layer component associated with each edge of $\bar{\Omega}$, and a corner layer associated with each corner of $\bar{\Omega}$. A similar decomposition is used even in the case of a single reaction-diffusion equation in 2 . In contrast, the analysis presented in the current paper needs no decomposition of $\boldsymbol{u}$ when investigating our scheme on a Bakhvalov mesh and only a relatively simple decomposition on a Shishkin mesh.

Assumption 1.1. Assume that the matrix $A$ is coercive, i.e., that there exists a constant $\gamma>0$ such that

$$
\boldsymbol{v}^{T} A \boldsymbol{v} \geq \gamma^{2} \boldsymbol{v}^{T} \boldsymbol{v} \text { in } \varnothing \quad \text { for all } \boldsymbol{v} \in \mathbb{R}^{m} .
$$

Assume also that for some $\alpha \in(0,1]$ we have $A \in C^{4, \alpha}(\bar{\Omega})^{M \times M}$ and $\boldsymbol{f} \in C^{4, \alpha}(\bar{\Omega})^{M}$, and that the restriction of $\boldsymbol{g}$ to each edge of $\bar{\Omega}$ yields a function lying in $C^{4, \alpha}[0,1]^{M}$ and that $\boldsymbol{g}$ is continuous at each of the corners; this implies that $\boldsymbol{g} \in C^{0, \alpha}(\partial \Omega)^{M}$. Finally, assume that

$$
-\varepsilon^{2} \boldsymbol{g}_{x x}(0+, 0)-\varepsilon^{2} \boldsymbol{g}_{y y}(0,0+)+A \boldsymbol{g}(0,0)=\boldsymbol{f}(0,0)
$$

with corresponding conditions at the other three corners of the domain $\bar{\Omega}$; these conditions can be loosely thought of as requiring " $\mathcal{L} \boldsymbol{g}=\boldsymbol{f}$ " at each corner .

Assumption 1.1 implies that (1.1) has a unique solution $\boldsymbol{u} \in C^{6, \alpha}(\Omega)^{M} \cap$ $C^{3, \alpha}(\bar{\Omega})^{M}$; see [5] and [4, Section 3]. Nevertheless, unlike [4], the operator $\mathcal{L}$ does not obey a maximum principle, and consequently our analysis is very different from that of [4].

Notation. Throughout the paper $C$, sometimes subscripted, is a generic positive constant that is independent of the perturbation parameter $\varepsilon$ and of the number of mesh intervals $N$. We set

$$
\begin{aligned}
|\boldsymbol{v}| & :=\sqrt{\boldsymbol{v}^{T} \boldsymbol{v}} \text { for } \boldsymbol{v} \in \mathbb{R}^{M} \\
\|v\|_{D} & :=\max _{(x, y) \in \bar{D}}|v(x, y)| \text { for } v \in C(\bar{D}), \\
\|\boldsymbol{v}\|_{D} & :=\max _{(x, y) \in \bar{D}}|\boldsymbol{v}(x, y)| \text { for } \boldsymbol{v} \in C(\bar{D})^{M},
\end{aligned}
$$

and we drop the subscript $D$ on these norms when $D=\varnothing$. The notation $\|\cdot\|_{D}$ is also used for the $L_{\infty}$ norms of continuous functions of one variable and for mesh-valued functions.

The notation $\partial^{m} w$ denotes any $m$ th-order partial derivative of a function $w$.

\section{Properties of the exact SOlution}

The following stability result, which is critical for our analysis, is a slight modification of an argument from [1]. Note how neatly it side-steps the absence of a maximum principle for $\mathcal{L}$.

Lemma 2.1. Let $\boldsymbol{w} \in C^{2}(\Omega)^{M} \cap C(\bar{\Omega})^{M}$. Then

$$
\|\boldsymbol{w}\| \leq \gamma^{-2}\|\mathcal{L} \boldsymbol{w}\|+\|\boldsymbol{w}\|_{\partial \Omega}
$$

Proof. Set $v=\frac{1}{2} \boldsymbol{w}^{T} \boldsymbol{w}$. Observe that

$$
-2 \boldsymbol{w}^{T} \Delta \boldsymbol{w}=-\Delta\left(\boldsymbol{w}^{T} \boldsymbol{w}\right)+2\left|\boldsymbol{w}_{x}\right|^{2}+2\left|\boldsymbol{w}_{y}\right|^{2} \geq-\Delta\left(\boldsymbol{w}^{T} \boldsymbol{w}\right) .
$$


Hence, taking the scalar product of $\boldsymbol{w}^{T}$ with $-\varepsilon^{2} \Delta \boldsymbol{w}+A \boldsymbol{w}=\mathcal{L} \boldsymbol{w}$ then invoking (1.2), we get

$$
-\varepsilon^{2} \Delta v+2 \gamma^{2} v \leq \boldsymbol{w}^{T} \mathcal{L} \boldsymbol{w} \quad \text { in } \Omega,
$$

and clearly $|v|_{\partial \Omega} \leq\|\boldsymbol{w}\|_{\partial \Omega}^{2} / 2$. Now the standard maximum principle for scalar problems posed on $\Omega$, with a constant barrier function, gives

$$
\|v\| \leq \frac{1}{2 \gamma^{2}}\|\boldsymbol{w}\|\|\mathcal{L} \boldsymbol{w}\|+\frac{1}{2}\|\boldsymbol{w}\|_{\partial \Omega}^{2}
$$

This implies that

$$
\|\boldsymbol{w}\|^{2}=2\|v\| \leq\|\boldsymbol{w}\|\left(\frac{\|\mathcal{L} \boldsymbol{w}\|}{\gamma^{2}}+\|\boldsymbol{w}\|_{\partial \Omega}\right)
$$

because $\|\boldsymbol{w}\|_{\partial \Omega} \leq\|\boldsymbol{w}\|$. The proposition of the lemma follows upon dividing by $\|\boldsymbol{w}\|$.

Thus $\mathcal{L}$ is $\left(L_{\infty}, L_{\infty}\right)$ stable even though it fails in general to satisfy a maximum principle.

Lemma 2.1 implies that (1.1) has a unique solution in $C^{0, \alpha}(\bar{\Omega})$, by the standard theory for solutions of systems of linear boundary-value problems [5, Chapter 7, Theorem 5.1], and this solution is bounded pointwise, uniformly in $\varepsilon$.

Corollary 2.1. The solution $\boldsymbol{u}$ of (1.1) satisfies the bound

$$
\|\boldsymbol{u}\| \leq \gamma^{-2}\|\boldsymbol{f}\|+\|\boldsymbol{g}\|_{\partial \Omega}
$$

This bound needs much less smoothness and compatibility of the data than is assumed in Assumption 1.1 .

In [4, Theorem 3.1] bounds on low-order derivatives of $\boldsymbol{u}$ are derived under hypotheses like Assumption 1.1 except that (1.2) is replaced by other assumptions on $A$. An inspection of the arguments in [4, Section 3] reveals that the key property needed there is an a priori $L_{\infty}(\Omega)$ bound like Corollary 2.1, which enables the invocation of Schauder estimates. Consequently, the same conclusions hold true for (1.1)-(1.2). That is, we have the following result:

Lemma 2.2. The solution $\boldsymbol{u}$ of (1.1) satisfies the bounds

$$
\left\|\partial^{k} \boldsymbol{u}\right\| \leq C \varepsilon^{-k} \quad \text { for } k=0, \ldots, 3
$$

furthermore,

$$
\left\|\partial_{x}^{2 j} \partial_{y}^{4-2 j} \boldsymbol{u}\right\| \leq C \varepsilon^{-4} \quad \text { for } j=0,1,2 .
$$

The inequality (2.1b), which stems from a result of Volkov [11, is somewhat surprising since in general one does not have $\boldsymbol{u} \in C^{4, \alpha}(\bar{\Omega})^{M}$; note that (2.1b) is valid only for certain fourth-order derivatives of $\boldsymbol{u}$.

We now derive sharper bounds on the pure derivatives of $\boldsymbol{u}$ that show that the large values seen in Lemma 2.2 do in fact decay rapidly as one moves away from $\partial \Omega$. 
Lemma 2.3. Let $\boldsymbol{u}$ be the solution of (1.1). Let $\varrho \in(0, \gamma)$ be arbitrary but fixed. Then there exists a constant $C$, which is independent of $\varepsilon$, such that

$$
\begin{aligned}
& \left|\partial_{x}^{k} \boldsymbol{u}(x, y)\right| \leq C\left[1+\varepsilon^{-k}\left(e^{-\varrho x / \varepsilon}+e^{-\varrho(1-x) / \varepsilon}\right)\right], \\
& \left|\partial_{y}^{k} \boldsymbol{u}(x, y)\right| \leq C\left[1+\varepsilon^{-k}\left(e^{-\varrho y / \varepsilon}+e^{-\varrho(1-y) / \varepsilon}\right)\right],
\end{aligned}
$$

for all $(x, y) \in \bar{\Omega}$ and $k=1, \ldots, 3$.

Proof. Fix $\varrho \in(0, \gamma)$ and set $B_{k}(x)=1+\varepsilon^{-k}\left(e^{-\varrho x / \varepsilon}+e^{-\varrho(1-x) / \varepsilon}\right)$. We use induction on $k$. For $k=0$ the lemma follows from Corollary 2.1. For $k=1,2,3$, differentiating (1.1) $k$ times with respect to $x$ gives

$$
-\varepsilon^{2} \Delta \partial_{x}^{k} \boldsymbol{u}+A \partial_{x}^{k} \boldsymbol{u}=\partial_{x}^{k} \boldsymbol{f}-\sum_{\ell=0}^{k-1}\left(\begin{array}{l}
k \\
l
\end{array}\right)\left(\partial_{x}^{k-\ell} A\right) \partial_{x}^{\ell} \boldsymbol{u}=: \boldsymbol{\varphi}_{k}
$$

$$
\text { with }\left|\boldsymbol{\varphi}_{k}(x, y)\right| \leq C B_{k-1}(x),
$$

where the bound on $\varphi_{k}$ is a consequence of the inductive hypothesis. Define $\tilde{\boldsymbol{u}}$ by $\partial_{x}^{k} \boldsymbol{u}=B_{k} \tilde{\boldsymbol{u}}$. Then

$$
-\varepsilon^{2} \Delta \tilde{\boldsymbol{u}}-2 \varepsilon^{2} \frac{B_{k}^{\prime}}{B_{k}} \partial_{x} \tilde{\boldsymbol{u}}+\left(A-\varepsilon^{2} \frac{B_{k}^{\prime \prime}}{B_{k}}\right) \tilde{\boldsymbol{u}}=\frac{\boldsymbol{\varphi}_{k}}{B_{k}} .
$$

Taking the inner product with $\tilde{\boldsymbol{u}}$ and setting $v_{k}=\frac{1}{2}|\tilde{\boldsymbol{u}}|^{2}$ we obtain, as in the proof of Lemma 2.1

$$
-\varepsilon^{2} \Delta v_{k}-2 \varepsilon^{2} \frac{B_{k}^{\prime}}{B_{k}} \partial_{x} v_{k}+2\left(\gamma^{2}-\varrho^{2}\right) v_{k} \leq C\|\tilde{\boldsymbol{u}}\|,
$$

by (1.2) and because $B_{k}^{\prime \prime}(x) \leq \varepsilon^{-2} \varrho^{2} B_{k}(x)$ and $\varphi_{k}(x, y) \leq C B_{k}(x)$. Boundary conditions for $v_{k}$ follow from Lemma 2.2. $\left|v_{k}\right| \leq C\|\tilde{\boldsymbol{u}}\|$ on $\partial \varnothing$. Application of a comparison principle for scalar equations yields

$$
\frac{1}{2}\|\tilde{\boldsymbol{u}}\|^{2}=\left\|v_{k}\right\| \leq C\|\tilde{\boldsymbol{u}}\| .
$$

Dividing by $\|\tilde{\boldsymbol{u}}\|$ gives $\|\tilde{\boldsymbol{u}}\| \leq C$; then, recalling the definition of $\tilde{\boldsymbol{u}}$, we obtain the first inequality of the lemma. The second inequality is proved in the same way.

Next we give an analogous bound for the pure fourth-order derivatives of $\boldsymbol{u}$, but the proof is different since these derivatives are discontinuous at the corners of the domain and consequently do not lie in $C(\bar{\Omega})$.

Lemma 2.4. Let $\boldsymbol{u}$ be the solution of (1.1). Let $\varrho \in(0, \gamma)$ be as in Lemma 2.3. Then there exists a constant $C$ such that

$$
\begin{aligned}
& \left|\partial_{x}^{4} \boldsymbol{u}(x, y)\right| \leq C\left[1+\varepsilon^{-4}\left(e^{-\varrho x / \varepsilon}+e^{-\varrho(1-x) / \varepsilon}\right)\right], \\
& \left|\partial_{y}^{4} \boldsymbol{u}(x, y)\right| \leq C\left[1+\varepsilon^{-4}\left(e^{-\varrho y / \varepsilon}+e^{-\varrho(1-y) / \varepsilon}\right)\right],
\end{aligned}
$$

for all $(x, y) \in \Omega$.

Proof. For each $(x, y) \in \Omega$, let $\Gamma(x, y)$ denote the distance from $(x, y)$ to the nearest of the four corners of $\Omega$. Set $\Omega_{\varepsilon}=\{(x, y) \in \Omega: \Gamma(x, y)<\varepsilon\}$. From (2.1b) we see immediately that (2.2a) holds true on $\bar{\Omega}_{\varepsilon}$, because the sum of exponentials in (2.2a) is then bounded below by a positive constant. But $\boldsymbol{u} \in C^{4, \alpha}\left(\overline{\Omega \backslash \Omega_{\varepsilon}}\right)^{M}$ since the corners of $\bar{\Omega}$ are excluded from $\overline{\Omega \backslash \Omega_{\varepsilon}}$; as regards boundary data for $\partial_{x}^{4} \boldsymbol{u}$ on 
$\partial\left(\Omega \backslash \Omega_{\varepsilon}\right)$, first (2.1b) implies that (2.2a) is valid on $\partial \Omega_{\varepsilon}$ and on $\left\{(x, y) \in \partial\left(\Omega \backslash \Omega_{\varepsilon}\right)\right.$ : $x=0$ or 1$\}$, then (1.1) gives $\left|\partial_{x}^{4} \boldsymbol{u}\right| \leq C$ on $\left\{(x, y) \in \partial\left(\Omega \backslash \Omega_{\varepsilon}\right): y=0\right.$ or 1$\}$. The maximum principle argument of Lemma 2.3 can now be invoked to prove (2.2a) on $\Omega \backslash \Omega_{\varepsilon}$.

The inequality (2.2b) follows similarly.

Note that the bounds of Lemmas 2.3 and 2.4 were obtained without constructing any decomposition of $u$.

Remark 2.1. The requirement that $\boldsymbol{g}$ be continuous at the corners of $\bar{\Omega}$ corresponds to zero-order compatibility of the data of (1.1), while the condition " $\mathcal{L} \boldsymbol{g}=\boldsymbol{f}$ " (see Assumption 1.1) at these corners corresponds to first-order compatibility. Compatibility conditions of order $k \geq 0$ are defined in [3, Section 3]: at the corner $(0,0)$, the $k$ th compatibility condition is

$$
\varepsilon^{2} \boldsymbol{g}_{s}^{(2 k)}(0)+(-1)^{k+1} \varepsilon^{2} \boldsymbol{g}_{w}^{(2 k)}(0)+\sum_{i=1}^{k}(-1)^{i-1} \partial_{x}^{2(k-i)} \partial_{y}^{2(i-1)} \boldsymbol{F}(0,0)=\mathbf{0},
$$

where $\boldsymbol{g}_{s}$ and $\boldsymbol{g}_{w}$ are the restrictions of $\boldsymbol{g}$ to $x=0$ and $y=0$ respectively, and $\boldsymbol{F}:=\boldsymbol{f}-\boldsymbol{A u}$. The compatibility conditions at the other corners of $\bar{\Omega}$ are analogous.

Let $\ell \geq 0$ be an integer. Similarly to Lemmas 2.3 and 2.4, one can prove that if $\boldsymbol{f} \in C^{2 \ell, \alpha}(\bar{\Omega})^{M}$, the restriction of $\boldsymbol{g}$ to each edge of $\bar{\Omega}$ yields a function lying in $C^{2 \ell+2, \alpha}[0,1]^{M}$, and the compatibility conditions up to order $\ell$ are satisfied at each corner, then for all $(x, y) \in \Omega$ one has

$$
\left|\partial_{x}^{k} \boldsymbol{u}(x, y)\right| \leq C\left[1+\varepsilon^{-k}\left(e^{-\varrho x / \varepsilon}+e^{-\varrho(1-x) / \varepsilon}\right)\right] \text { for } k=0,1, \ldots, 2 \ell+2,
$$

with an analogous result for $\partial_{y}^{k} \boldsymbol{u}(x, y)$.

Remark 2.2. Consider the case of a single equation, i.e., $M=1$. Then one can apply a maximum principle argument directly to (1.1), i.e., most of the argument of Lemma 2.1 can be discarded, and following our subsequent analysis one obtains again the bounds of Lemmas 2.3 and 2.4. These bounds are sharper than the bounds obtained in [2] via a lengthy decomposition of $\boldsymbol{u}$. Our bounds are identical to those stated in [6], but the proof there is flawed.

Furthermore, when our arguments are applied to a reaction-diffusion system posed on a one-dimensional domain, this yields an improvement of the a priori bounds of [1].

\section{Discretization}

Consider an arbitrary tensor-product mesh $\bar{\omega}=\bar{\omega}_{x} \times \bar{\omega}_{y}$ on $\bar{\Omega}$, with $\bar{\omega}_{x}: 0=$ $x_{0}<x_{1}<\cdots<x_{N}=1$ and $\bar{\omega}_{y}: 0=y_{0}<y_{1}<\cdots<y_{N}=1$. Let $\omega=\bar{\omega} \cap \Omega$ and $\partial \omega=\bar{\omega} \cap \partial \Omega$. Set $h_{i}:=x_{i}-x_{i-1}$ and $k_{j}:=y_{j}-y_{j-1}$. Given a mesh function $\left\{v_{i, j}\right\}_{i, j=0}^{N}$, introduce the standard difference operators

$$
\delta_{x}^{2} v_{i, j}:=\frac{1}{\hbar_{i}}\left(\frac{v_{i+1, j}-v_{i, j}}{h_{i+1}}-\frac{v_{i, j}-v_{i-1, j}}{h_{i}}\right) \quad \text { for } i, j=1, \ldots, N-1,
$$

and

$$
\delta_{y}^{2} v_{i, j}:=\frac{1}{k_{j}}\left(\frac{v_{i, j+1}-v_{i, j}}{k_{j+1}}-\frac{v_{i, j}-v_{i, j-1}}{k_{j}}\right) \quad \text { for } \quad i, j=1, \ldots, N-1,
$$


where

$$
\hbar_{i}:=\frac{h_{i+1}+h_{i}}{2} \text { and } \quad k_{j}:=\frac{k_{j+1}+k_{j}}{2} .
$$

For vector-valued mesh functions $\boldsymbol{v}$ we use the notation

$$
\delta_{x}^{2} \boldsymbol{v}=\left(\delta_{x}^{2} v_{1}, \ldots, \delta_{x}^{2} v_{M}\right)^{T} \quad \text { and } \quad \delta_{y}^{2} \boldsymbol{v}=\left(\delta_{y}^{2} v_{1}, \ldots, \delta_{y}^{2} v_{M}\right)^{T} .
$$

Then our discretization is: Find $\boldsymbol{U}$ such that

$$
\begin{aligned}
{[\boldsymbol{L} \boldsymbol{U}]_{i, j}:=-\varepsilon^{2}\left(\delta_{x}^{2} \boldsymbol{U}+\delta_{y}^{2} \boldsymbol{U}\right)_{i, j}+A\left(x_{i}, y_{j}\right) \boldsymbol{U}_{i, j}=\boldsymbol{f}_{i, j} } & \text { in } \omega \\
\boldsymbol{U}_{i, j}=\boldsymbol{g}_{i, j} & \text { on } \partial \omega .
\end{aligned}
$$

Lemma 3.1. The discrete operator $\boldsymbol{L}$ satisfies the stability inequality

$$
\|\boldsymbol{W}\|_{\bar{\omega}} \leq \gamma^{-2}\|\boldsymbol{L} \boldsymbol{W}\|_{\omega}+\|\boldsymbol{W}\|_{\partial \omega}
$$

for arbitrary vector-valued functions $\boldsymbol{W}$ defined on $\bar{\omega}$.

Proof. This proof is a discrete analogue of the argument for Lemma 2.1. Let $V=$ $\frac{1}{2} \boldsymbol{W}^{T} \boldsymbol{W}$. Note that

$$
\boldsymbol{W}_{i, j}^{T} \delta_{x}^{2} \boldsymbol{W}_{i, j}=\frac{1}{2} \delta_{x}^{2}\left(\boldsymbol{W}^{T} \boldsymbol{W}\right)_{i, j}-\frac{1}{2 \hbar_{i}}\left(\frac{\left|\boldsymbol{W}_{i+1, j}-\boldsymbol{W}_{i, j}\right|^{2}}{h_{i+1}}+\frac{\left|\boldsymbol{W}_{i, j}-\boldsymbol{W}_{i-1, j}\right|^{2}}{h_{i}}\right)
$$

with a similar identity for $\delta_{y}^{2}$. Thus

$$
\boldsymbol{W}_{i, j}^{T}\left(\delta_{x}^{2}+\delta_{y}^{2}\right) \boldsymbol{W}_{i, j} \geq\left(\delta_{x}^{2}+\delta_{y}^{2}\right) V_{i, j} .
$$

Hence, using (1.2), we get $-\varepsilon^{2}\left(\delta_{x}^{2}+\delta_{y}^{2}\right) V+2 \gamma^{2} V \leq \boldsymbol{W}^{T} \boldsymbol{L} \boldsymbol{W}$ on $\omega$, and $|V|_{\partial \omega} \leq$ $\frac{1}{2}\|\boldsymbol{W}\|_{\partial \omega}^{2}$. A standard discrete maximum principle for scalar problems, with a constant barrier function, yields

$$
\|V\|_{\bar{\omega}} \leq \frac{1}{2 \gamma^{2}}\|\boldsymbol{W}\|_{\omega}\|\boldsymbol{L} \boldsymbol{W}\|_{\omega}+\frac{1}{2}\|\boldsymbol{W}\|_{\partial \omega}^{2} .
$$

Hence

$$
\|\boldsymbol{W}\|_{\bar{\omega}}^{2}=2\|V\|_{\bar{\omega}} \leq\|\boldsymbol{W}\|_{\bar{\omega}}\left(\frac{\|\boldsymbol{L} \boldsymbol{W}\|_{\omega}}{\gamma^{2}}+\|\boldsymbol{W}\|_{\partial \omega}\right) .
$$

The proposition of the lemma follows.

Lemma 3.1 implies that the linear system (3.1) has a unique solution $\boldsymbol{U}$.

3.1. Layer-adapted meshes. We shall consider two types of a priori meshes: Bakhvalov and Shishkin meshes. When constructing these meshes, some a priori knowledge of the behaviour of the derivatives of the exact solution is essential. This information is provided by Lemmas 2.3 and 2.4 . Let $\varrho \in(0, \gamma)$ be arbitrary but fixed. Then

$$
\left|\partial_{x}^{k} \boldsymbol{u}(x, y)\right| \leq C\left[1+\varepsilon^{-k}\left(e^{-\varrho x / \varepsilon}+e^{-\varrho(1-x) / \varepsilon}\right)\right] \text { for } k=0, \ldots, 4,
$$

with analogous bounds for $\partial_{y}^{k} \boldsymbol{u}$. 
Bakhvalov meshes [1]: These meshes are based on user-chosen mesh parameters $\sigma>0$ and $q \in(0,1 / 2)$, where the grid points are $x_{i}=i / N$ if $\sigma \varepsilon \geq \varrho q$, while when $\sigma \varepsilon<\varrho q$ one sets

$$
x_{i}= \begin{cases}\varphi(i / N) & \text { for } i \leq N / 2, \\ 1-\varphi((N-i) / N) & \text { for } i>N / 2,\end{cases}
$$

with a mesh generating function $\varphi$ defined by

$$
\varphi(\xi)=\left\{\begin{array}{lll}
\chi(\xi):=-\frac{\sigma \varepsilon}{\varrho} \ln \left(1-\frac{\xi}{q}\right) & \text { for } & \xi \in[0, \tau], \\
\pi(\xi):=\chi(\tau)+\chi^{\prime}(\tau)(\xi-\tau) & \text { for } & \xi \in[\tau, 1 / 2] .
\end{array}\right.
$$

The transition point $\tau$ is determined by the equation $(1-2 \tau) \chi^{\prime}(\tau)=1-2 \chi(\tau)$. Thus the tangent to the graph of $\chi$ at $(\tau, \chi(\tau))$ passes through $(1 / 2,1 / 2)$. This defines the mesh on $[0,1 / 2]$ and it is extended to $[0,1]$ by reflection about $x=1 / 2$. The resulting mesh generating function $\varphi$ lies in $C^{1}[0,1]$.

Shishkin meshes [9, 10]: These meshes are constructed as follows. Choose mesh parameters $\sigma>0$ and $q \in(0,1 / 2)$. Define the transition point

$$
\lambda:=\min \left\{q, \frac{\sigma \varepsilon}{\varrho} \ln N\right\} .
$$

Assuming that $q N$ is an integer, we divide each of the two intervals $[0, \lambda]$ and $[1-\lambda, 1]$ uniformly into $q N$ subintervals and $[\lambda, 1-\lambda]$ into $(1-2 q) N$ subintervals of equal length. A typical choice would be $q=1 / 4$ and $N$ divisible by 4 . The mesh generating function for a Shishkin mesh is piecewise linear and continuous.

3.2. Error analysis. In the layer regions the Bakhvalov mesh is not approximately equidistant. Consequently, the truncation error of the difference scheme is apparently only first order at points in the layers, but a more delicate analysis given in 1 f for a two-point boundary value problem shows that the truncation error at every mesh point is in fact $\mathcal{O}\left(N^{-2}\right)$ uniformly in $\varepsilon$. It is straightforward to modify Bakhvalov's analysis for the case of (1.1); we shall outline how this is done in an appendix because, while [1] is often cited as a seminal paper, its contents are not widely known and because in recent publications Bakhvalov meshes have received much less attention than Shishkin meshes even though they give more accurate numerical solutions.

The analysis of singularly perturbed reaction-diffusion problems on Shishkin meshes on the domain $\Omega$ has been carried out by Clavero et al. 2] in the case of a single equation and by Kellogg et al. 4 for a system of equations, but the error analysis in both papers relies on the lengthy construction of a decomposition of the solution. The analysis for Shishkin meshes in the present paper is much simpler.

Theorem 3.1. Let Assumption 1.1 be satisfied. Assume that the mesh parameter $\sigma$ satisfies $\sigma \geq 2$. Then

$$
\|\boldsymbol{u}-\boldsymbol{U}\|_{\bar{\omega}} \leq \begin{cases}C N^{-2} & \text { for Bakhvalov meshes } \\ C N^{-2} \ln ^{2} N & \text { for Shishkin meshes. }\end{cases}
$$

Proof. Let $\boldsymbol{\eta}=\boldsymbol{u}-\boldsymbol{U}$ denote the error. Lemma 3.1 yields

$$
\|\boldsymbol{u}-\boldsymbol{U}\|_{\bar{\omega}} \leq \gamma^{-2}\|\boldsymbol{L}(\boldsymbol{u}-\boldsymbol{U})\|_{\omega}=\gamma^{-2}\|\boldsymbol{L} \boldsymbol{u}-\mathcal{L} \boldsymbol{u}\|_{\omega}
$$


because $\boldsymbol{u}=\boldsymbol{U}$ on $\partial \omega$. For the $m$ th component of the truncation error $\boldsymbol{L} \boldsymbol{\eta}=$ $\boldsymbol{L u}-\mathcal{L} \boldsymbol{u}$ we have

$$
(\boldsymbol{L} \boldsymbol{u})_{m}-(\mathcal{L} \boldsymbol{u})_{m}=\varepsilon^{2}\left(\partial_{x}^{2} u_{m}-\delta_{x}^{2} u_{m}\right)+\varepsilon^{2}\left(\partial_{y}^{2} u_{m}-\delta_{y}^{2} u_{m}\right) \text { for } m=1, \ldots, M
$$

For any $\psi \in C^{4}(\Omega)$ with $\partial_{x}^{k} \psi$ bounded on $\bar{\Omega}$ for $k=0, \ldots, 4$, Taylor expansions show that

$$
\left|\left[\partial_{x}^{2} \psi-\delta_{x}^{2} \psi\right]_{i, j}\right| \leq\left\{\begin{array}{l}
C\left\|\partial_{x}^{2} \psi\left(\cdot, y_{j}\right)\right\|_{\left[x_{i-1}, x_{i+1}\right]} \\
C\left(h_{i}+h_{i+1}\right)\left\|\partial_{x}^{3} \psi\left(\cdot, y_{j}\right)\right\|_{\left[x_{i-1}, x_{i+1}\right]} \\
\left|h_{i}-h_{i+1}\right|\left|\partial_{x}^{3} \psi\left(x_{i}, y_{j}\right)\right| \\
+C\left(h_{i}+h_{i+1}\right)^{2}\left\|\partial_{x}^{4} \psi\left(\cdot, y_{j}\right)\right\|_{\left[x_{i-1}, x_{i+1}\right]}
\end{array}\right.
$$

There are analogous bounds for $\partial_{y}^{2} \psi-\delta_{y}^{2} \psi$.

Bakhvalov meshes. By choosing $\sigma \geq 2$, an application of the technique in 1 for one-dimensional problems, combined with the bound (3.2) on $\partial_{x}^{k} u_{m}$ for $k=2,3,4$, yields

$$
\varepsilon^{2}\left|\left(\partial_{x}^{2} u_{m}-\delta_{x}^{2} u_{m}\right)_{i, j}\right| \leq C N^{-2} \text { for } i, j=1, \ldots, N-1 \text { and } m=1, \ldots, M ;
$$

see the Appendix for details. There is a corresponding bound for $\partial_{y}^{2} u_{m}-\delta_{y}^{2} u_{m}$. Thus the truncation error is uniformly bounded by $C N^{-2}$. Invoking (3.3), the proof of Theorem 3.1 for the Bakhvalov mesh is complete.

Shishkin meshes. First consider the case where $\lambda=q \leq \sigma \varepsilon \varrho^{-1} \ln N$. Then the mesh is uniform with mesh size $N^{-1}$. Furthermore, $\varepsilon^{-1} \leq C \ln N$. Hence (3.2) and (3.4c) give $\|\boldsymbol{L u}-\mathcal{L} \boldsymbol{u}\|_{\omega} \leq C N^{-2} \ln ^{2} N$. Invoking (3.3), Theorem 3.1 follows in this case.

Now suppose that $\lambda=\sigma \varepsilon \varrho^{-1} \ln N \leq q$. Let $x^{*}=2 \varepsilon \varrho^{-1} \ln (1 / \varepsilon)$. For each $m \in\{1, \ldots, M\}$ and $(x, y) \in \bar{\Omega}$, set

$$
v_{m}(x, y)= \begin{cases}\sum_{\nu=0}^{4} \frac{\left(x-x^{*}\right)^{\nu}}{\nu !} \partial_{x}^{\nu} u_{m}\left(x^{*}, y\right) & \text { for } 0 \leq x \leq x^{*} \\ u_{m}(x, y) & \text { for } x^{*} \leq x \leq 1-x^{*}, \\ \sum_{\nu=0}^{4} \frac{\left(x-x^{*}\right)^{\nu}}{\nu !} \partial_{x}^{\nu} u_{m}\left(1-x^{*}, y\right) & \text { for } 1-x^{*} \leq x \leq 1,\end{cases}
$$

and $w_{m}(x, y)=u_{m}(x, y)-v(x, y)$. Then Lemmas 2.3 and 2.4 and the choice of $x^{*}$ imply that

$$
\begin{gathered}
\left|\partial_{x}^{k} v_{m}(x, y)\right| \leq C\left(1+\varepsilon^{2-k}\right) \text { and } \\
\left|\partial_{x}^{k} w_{m}(x, y)\right| \leq C \varepsilon^{-k}\left(e^{-\varrho x / \varepsilon}+e^{-\varrho(1-x) / \varepsilon}\right) \text { for } 0 \leq k \leq 4
\end{gathered}
$$

cf. 7. That is, $\boldsymbol{u}=\boldsymbol{v}+\boldsymbol{w}$, i.e., we have decomposed $\boldsymbol{u}$ into a sum of a regular component $\boldsymbol{v}$ and a layer component $\boldsymbol{w}=\left(w_{1}, \ldots, w_{m}\right)^{T}$. We remark that our decomposition does not in general satisfy $\mathcal{L} \boldsymbol{v}=\boldsymbol{f}$ and $\mathcal{L} \boldsymbol{w}=\mathbf{0}$; these additional properties, which are not needed here, have been obtained for various singular perturbation problems via more complicated analyses.

Split the truncation error by writing

$$
\left[\partial_{x}^{2} u_{m}-\delta_{x}^{2} u_{m}\right]_{i, j}=\left[\partial_{x}^{2} v_{m}-\delta_{x}^{2} v_{m}\right]_{i, j}+\left[\partial_{x}^{2} w_{m}-\delta_{x}^{2} w_{m}\right]_{i, j} .
$$

When bounding the truncation error in $\boldsymbol{v}$ use (3.4b) for $i=q N$ or $i=(1-q) N$, i.e., at the mesh transition points, and at other points use (3.4c). For the layer 
term $\boldsymbol{w}$, invoke (3.4a) for $i=q N, \ldots,(1-q) N$ and (3.4c) otherwise. This yields

$$
\varepsilon^{2}\left|\left[\partial_{x}^{2} u_{m}-\delta_{x}^{2} u_{m}\right]_{i, j}\right| \leq C N^{-2} \ln ^{2} N+ \begin{cases}C \varepsilon N^{-1} & \text { for } i \in\{q N,(1-q) N\}, \\ 0 & \text { otherwise. }\end{cases}
$$

We cannot invoke (3.3) to get the desired error bound. Instead we proceed as follows. Write $\boldsymbol{\eta}=\boldsymbol{\eta}_{1}+\boldsymbol{\eta}_{2}$, where $\boldsymbol{\eta}_{1}$ and $\boldsymbol{\eta}_{2}$ are the solutions of

$$
\begin{array}{llll}
\boldsymbol{L} \boldsymbol{\eta}_{1}=\varepsilon^{2}\left(\partial_{x}^{2} \boldsymbol{u}-\delta_{x}^{2} \boldsymbol{u}\right) & \text { in } \omega, & \boldsymbol{\eta}_{1}=\mathbf{0} & \text { on } \partial \omega, \\
\boldsymbol{L} \boldsymbol{\eta}_{2}=\varepsilon^{2}\left(\partial_{y}^{2} \boldsymbol{u}-\delta_{y}^{2} \boldsymbol{u}\right) & \text { in } \omega, & \boldsymbol{\eta}_{2}=\mathbf{0} & \text { on } \partial \omega .
\end{array}
$$

In order to bound $\boldsymbol{\eta}_{1}$ we use the technique of Lemma 3.1. Set $V=\frac{1}{2} \boldsymbol{\eta}_{1}^{T} \boldsymbol{\eta}_{1}$. Then

$$
-\varepsilon^{2}\left(\delta_{x}^{2}+\delta_{y}^{2}\right) V+2 \gamma^{2} V \leq C\left\|\boldsymbol{\eta}_{1}\right\|\left|\boldsymbol{L} \boldsymbol{\eta}_{1}\right| \quad \text { in } \varnothing, \quad V=0 \quad \text { on } \partial \varnothing .
$$

One can apply a discrete comparison principle for scalar equations using a barrier function from 8 :

$$
\bar{V}_{i, j}=C_{1}\left\|\boldsymbol{\eta}_{1}\right\| N^{-2}\left(\ln ^{2} N+\lambda \varepsilon^{-1} \varphi_{i}\right)
$$

where

$$
\varphi_{i}:= \begin{cases}x_{i} \lambda^{-1} & \text { for } i=0, \ldots, q N \\ 1 & \text { for } i=q N, \ldots,(1-q) N \\ \left(1-x_{i}\right) \lambda^{-1} & \text { for } i=(1-q) N, \ldots, N .\end{cases}
$$

Then with $C_{1}$ chosen sufficiently large, independently of $\varepsilon$, one obtains $\|V\|_{\bar{\omega}} \leq$ $C\|\bar{V}\|_{\bar{\omega}}$ and hence $\left\|\boldsymbol{\eta}_{1}\right\| \leq C N^{-2} \ln ^{2} N$. An identical bound is obtained for $\boldsymbol{\eta}_{2}$. The assertion of the theorem follows for the Shishkin mesh.

3.3. Numerical results. We now present the results of some numerical experiments in order to illustrate the conclusions of Theorem 3.1, and to check if they are sharp.

\section{Example 1.}

$$
\begin{aligned}
-\varepsilon^{2} \Delta u_{1}+2 u_{1}+x u_{2} & =\sin (\pi(x+y)) & & \text { in } \varnothing,\left.u_{1}\right|_{\partial \varnothing}=0 \\
-\varepsilon^{2} \Delta u_{2}+\left(1+y^{2}\right) u_{1}+(3+x) u_{2} & =3 x(1-x)+y(1-y) & & \text { in } \varnothing,\left.u_{1}\right|_{\partial \varnothing}=0
\end{aligned}
$$

For this problem $\gamma^{2} \approx 1.12$.

\section{Example 2.}

$$
\begin{aligned}
-\varepsilon^{2} \Delta u_{1}+(3+x) u_{1}+y u_{2}+x^{2} u_{3} & =\sin (\pi(x+y)) & & \text { in } \varnothing,\left.u_{1}\right|_{\partial \varnothing}=0, \\
-\varepsilon^{2} \Delta u_{2}+y u_{1}+(4-y) u_{2}+x y u_{3} & =0 & & \text { in } \varnothing,\left.u_{2}\right|_{\partial \varnothing}=0, \\
-\varepsilon^{2} \Delta u_{3}+x^{2} u_{1}+x y u_{2}+\left(3-x^{2}\right) u_{3} & =e^{x} y(1-y) & & \text { in } \varnothing,\left.u_{3}\right|_{\partial \varnothing}=0,
\end{aligned}
$$

with $\gamma^{2} \approx 1.32$. For both problems we take $\varrho=1$ and $\sigma=2$ in the construction of the meshes.

The exact solutions to both test problems is not available, so we estimate the accuracy of the numerical solution by comparing it with the numerical solution obtained from Richardson extrapolation over two meshes, which has a higher order of accuracy. Thus, let $\boldsymbol{U}_{\varepsilon}^{N}$ be the solution of the difference scheme on the original 
Shishkin mesh and $\tilde{\boldsymbol{U}}_{\varepsilon}^{2 N}$ on the mesh obtained by uniformly bisecting the original mesh. Then the extrapolated solution is

$$
\boldsymbol{U}_{\varepsilon}^{R, N}:=\frac{4 \tilde{\boldsymbol{U}}_{\varepsilon}^{2 N}-\boldsymbol{U}_{\varepsilon}^{N}}{3} .
$$

We then compute the error

$$
\eta_{\varepsilon}^{N}:=\left\|\boldsymbol{U}_{\varepsilon}^{N}-\boldsymbol{U}_{\varepsilon}^{R, N}\right\|_{\omega}=\frac{4}{3}\left\|\boldsymbol{U}_{\varepsilon}^{N}-\tilde{\boldsymbol{U}}_{\varepsilon}^{2 N}\right\|_{\omega}
$$

for various values of $\varepsilon$.

We estimate the uniform errors for a fixed $N$ by taking the maximum error over a wide range of $\varepsilon$, namely

$$
\eta^{N}:=\max _{\varepsilon^{2}=1,10^{-1}, \ldots, 10^{-12}} \eta_{\varepsilon}^{N} .
$$

The results of our test computations are displayed in the following table. As well as the uniform errors $\eta^{N}$, we also give experimental rates of convergence $r^{N}$ which are computed by means of the formula

$$
r^{N}=\log _{2}\left(\eta^{N} / \eta^{2 N}\right)
$$

We also estimate the constants in the error estimate, i. e., if we have the theoretical error bound $\eta^{N} \leq C \vartheta(N)$, then we compute the quantity $C^{N}=\eta^{N} / \vartheta(N)$.

The numerical results are in accordance with Theorem 3.1 and illustrate its sharpness.

\begin{tabular}{c|ccc|ccc||ccc|ccc} 
& \multicolumn{6}{|c||}{ Example 1 } & \multicolumn{8}{c}{ Example 2 } \\
\hline & \multicolumn{3}{|c|}{ Shishkin mesh } & \multicolumn{2}{c||}{ Bakhvalov mesh } & \multicolumn{2}{c}{ Shishkin mesh } & \multicolumn{2}{c}{ Bakhvalov mesh } \\
\hline$N$ & $\eta^{N}$ & $r^{N}$ & $C^{N}$ & $\eta^{N}$ & $r^{N}$ & $C^{N}$ & $\eta^{N}$ & $r^{N}$ & $C^{N}$ & $\eta^{N}$ & $r^{N}$ & $C^{N}$ \\
\hline 16 & $1.45 \mathrm{e}-2$ & 0.93 & 0.48 & $5.17 \mathrm{e}-3$ & 1.81 & 1.32 & $1.18 \mathrm{e}-2$ & 0.80 & 0.39 & $4.53 \mathrm{e}-3$ & 1.82 & 1.16 \\
32 & $7.57 \mathrm{e}-3$ & 1.41 & 0.65 & $1.37 \mathrm{e}-3$ & 1.94 & 1.40 & $6.47 \mathrm{e}-3$ & 1.20 & 0.55 & $1.19 \mathrm{e}-3$ & 1.95 & 1.22 \\
64 & $2.84 \mathrm{e}-3$ & 1.47 & 0.67 & $3.52 \mathrm{e}-4$ & 1.99 & 1.44 & $2.82 \mathrm{e}-3$ & 1.46 & 0.67 & $3.03 \mathrm{e}-4$ & 1.98 & 1.24 \\
128 & $1.02 \mathrm{e}-3$ & 1.58 & 0.71 & $8.86 \mathrm{e}-5$ & 2.00 & 1.45 & $1.02 \mathrm{e}-3$ & 1.58 & 0.71 & $7.61 \mathrm{e}-5$ & 2.00 & 1.25 \\
256 & $3.43 \mathrm{e}-4$ & 1.65 & 0.73 & $2.22 \mathrm{e}-5$ & 2.00 & 1.45 & $3.41 \mathrm{e}-4$ & 1.65 & 0.73 & $1.90 \mathrm{e}-5$ & 2.00 & 1.25 \\
512 & $1.09 \mathrm{e}-4$ & - & 0.73 & $5.55 \mathrm{e}-6$ & - & 1.45 & $1.09 \mathrm{e}-4$ & - & 0.73 & $4.76 \mathrm{e}-6$ & - & 1.25
\end{tabular}

\section{Appendix: Truncation error on the BakHValov Mesh}

In this appendix we reproduce the argument from [1] to prove the truncation error bound that was used in the proof of Theorem 3.1.

When $\sigma \varepsilon \geq \varrho q$ the mesh is uniform with mesh size $N^{-1}$. Furthermore, $\varepsilon^{-1} \leq C$. Thus

$$
\varepsilon^{2}\left\|\partial_{x}^{2} u_{m}-\delta_{x}^{2} u_{m}\right\|_{\omega} \leq C N^{-2}
$$

by (3.2) and (3.4c).

Now consider the case $\sigma \varepsilon<\varrho q$. For simplicity we consider only the layer at $x=0$ and assume that $x_{i}=\varphi\left(t_{i}\right) \leq 1 / 2$.

From the construction of $\varphi$ one must have $\tau<q$ and it follows that $1<\chi^{\prime}(\tau)<\tilde{q}$, where we set $\tilde{q}=1 /(1-2 q)$. Define the auxiliary points $\tau_{1}, \tau_{2}$ in $(0, q)$ by $\chi^{\prime}\left(\tau_{1}\right)=\tilde{q}$ and $\chi^{\prime}\left(\tau_{2}\right)=1$. Then $\tau_{2}=q-\sigma \varepsilon / \varrho<\tau<\tau_{1}=q-\sigma \varepsilon(1-2 q) / \varrho$ because $\chi^{\prime \prime}>0$ on $[0, q)$.

(i) $\varphi^{\prime}(t) \leq \chi^{\prime}(\tau) \leq \tilde{q}$ for $t \in[0,1]$. Thus,

$$
h_{i}=\int_{t_{i-1}}^{t_{i}} \varphi^{\prime}(t) d t \leq \tilde{q} N^{-1} \quad \text { for } \quad i=1, \ldots, N .
$$


(ii) For $t \leq t_{i}<q$ we have $\varphi^{\prime}(t) \leq \chi^{\prime}(t)=\sigma \varepsilon / \varrho(q-t) \leq \sigma \varepsilon / \varrho\left(q-t_{i}\right)$. Hence,

$$
\begin{aligned}
h_{i} & =\int_{t_{i-1}}^{t_{i}} \varphi^{\prime}(t) d t \leq N^{-1} \varphi^{\prime}\left(t_{i}\right) \leq \frac{\sigma \varepsilon}{\varrho N\left(q-t_{i}\right)} \\
& \leq \frac{2 \sigma \varepsilon}{\varrho N\left(q-t_{i-1}\right)} \quad \text { for } t_{i} \leq q-N^{-1} .
\end{aligned}
$$

(iii) $h_{i+1}-h_{i}=x_{i+1}-2 x_{i}+x_{i-1}=\varphi^{\prime \prime}\left(t_{i}^{*}\right) N^{-2}$ for some $t_{i}^{*} \in\left[t_{i-1}, t_{i+1}\right]$. Now

$$
\varphi^{\prime \prime}(t) \leq \chi^{\prime \prime}(\tau)=\frac{\sigma \varepsilon}{\varrho(q-\tau)^{2}} \quad \text { and } \quad \frac{1}{q-\tau} \leq \frac{1}{q-\tau_{1}}=\frac{\varrho \tilde{q}}{\sigma \varepsilon},
$$

which gives

$$
\left|h_{i+1}-h_{i}\right| \leq \frac{\varrho \tilde{q}^{2}}{\sigma \varepsilon} N^{-2} .
$$

Furthermore, we have the bound

$$
\varphi^{\prime \prime}\left(t_{i}^{*}\right) \leq \frac{\sigma \varepsilon}{\varrho\left(q-t_{i+1}\right)^{2}} \leq \frac{4 \sigma \varepsilon}{\varrho\left(q-t_{i}\right)^{2}} \quad \text { for } \quad t_{i} \leq q-\frac{2}{N}
$$

which yields

$$
\left|h_{i+1}-h_{i}\right| \leq \frac{4 \sigma \varepsilon}{N^{2} \varrho\left(q-t_{i}\right)^{2}} \quad \text { for } \quad t_{i} \leq q-\frac{2}{N} .
$$

and

$$
e^{-\varrho x_{i} / \varepsilon}=\left(\frac{q-t_{i}}{q}\right)^{\sigma} \quad \text { for } t_{i} \leq \tau
$$

Henceforth, let $\sigma \geq 2$. Using (3.4c), (3.2), (A.2) and (A.8), we get

$$
\varepsilon^{2}\left|\left[\partial_{x}^{2} u_{m}-\delta_{x}^{2} u_{m}\right]_{i, j}\right| \leq C N^{-2} \text { for } \tau_{2} \leq t_{i-1}
$$

which is the region outside the layer. For $t_{i} \leq q-2 N^{-1}$ (the layer region), from $(3.4 \mathrm{c})$ and (3.2) one arrives at

$$
\begin{aligned}
\varepsilon^{2}\left|\left[\partial_{x}^{2} u_{m}-\delta_{x}^{2} u_{m}\right]_{i, j}\right| \leq C \mid & h_{i}-h_{i+1}\left|\varepsilon^{2}+C\right| h_{i}-h_{i+1} \mid \varepsilon^{-1} e^{-\varrho x_{i} / \varepsilon} \\
& +C\left(h_{i}+h_{i+1}\right)^{2} \varepsilon^{2}+C\left(h_{i}+h_{i+1}\right)^{2} \varepsilon^{-2} e^{-\varrho x_{i-1} / \varepsilon} .
\end{aligned}
$$

To bound the first term on the right-hand side use (A.4); for the second term, use (A.6) and (A.7); for the third term, use (A.2); and for the fourth, use (A.3), (A.7) and $q-t_{i-1} \leq 3\left(q-t_{i}\right) / 2$. This yields

$$
\varepsilon^{2}\left|\left[\partial_{x}^{2} u_{m}-\delta_{x}^{2} u_{m}\right]_{i, j}\right| \leq C N^{-2} \quad \text { for } \quad t_{i} \leq q-2 N^{-1} .
$$

We are left with the transition region where $\tau_{2}>t_{i-1}$ and $t_{i}>q-2 N^{-1}$. Thus,

$$
q-\frac{2}{N}<t_{i}<\tau_{2}+\frac{1}{N}=q-\frac{\sigma \varepsilon}{\varrho}+\frac{1}{N}<q+\frac{1}{N} .
$$


Observe that the first two inequalities here imply that $\varepsilon<3 \varrho /(\sigma N)$. Use (3.4a):

$$
\varepsilon^{2}\left|\left[\partial_{x}^{2} u_{m}-\delta_{x}^{2} u_{m}\right]_{i, j}\right| \leq C\left(\varepsilon^{2}+e^{-\varrho x_{i-1} / \varepsilon}\right) \leq C N^{-2},
$$

by (A.8) and $\varepsilon \leq C N^{-1}$.

Thus, on a Bakhvalov mesh, the truncation error in the maximum norm is bounded uniformly by $\mathcal{O}\left(N^{-2}\right)$.

\section{ACKNOWLEDGEMENT}

The numerical results were provided by Niall Madden, whose willing help the authors gratefully acknowledge.

\section{REFERENCES}

[1] N. S. Bakhvalov. On the optimization of methods for solving boundary value problems with boundary layers (in Russian). Zh. Vychisl. Mat. i Mat. Fis., 9:841-859, 1969. MR0255066 (40:8273)

[2] C. Clavero, J. L. Gracia, and E. O'Riordan. A parameter robust numerical method for a two dimensional reaction-diffusion problem. Math. Comp., 74:1743-1758, 2005. MR2164094 (2006e:65192)

[3] H. Han and R. B. Kellogg. Differentiability properties of solutions of the equation $-\epsilon^{2} \Delta u+$ $r u=f(x, y)$ in a square. SIAM J. Math. Anal., 21:394-408, 1990. MR1038899(91e:35025)

[4] R. B. Kellogg, N. Madden, and M. Stynes. A parameter-robust numerical method for a system of reaction-diffusion equations in two dimensions. Numer. Meth. Partial Diff. Equations, 2007. (to appear).

[5] O. A. Ladyzhenskaya and N. N. Uraltseva. Linear and quasilinear elliptic equations. Translated from the Russian by Scripta Technica, Inc., Translation editor: Leon Ehrenpreis. Academic Press, New York, 1968. MR0244627 (39:5941)

[6] J. Li and I. M. Navon. Uniformly convergent finite element methods for singularly perturbed elliptic boundary value problems. I. Reaction-diffusion type. Comput. Math. Appl., 35:57-70, 1998. MR 1605555 (98m:65193)

[7] T. Linß. The necessity of Shishkin-decompositions. Appl. Math. Lett., 14:891-896, 2001. MR.1849244

[8] J. J. H. Miller, E. O'Riordan, G. I. Shishkin, and L. P. Shishkina. Fitted mesh methods for problems with parabolic boundary layers. Math. Proc. R. Ir. Acad., 98A:173-190, 1998. MR.1759430 (2001e:65126)

[9] H.-G. Roos, M. Stynes, and L. Tobiska. Numerical methods for singularly perturbed differential equations, volume 24 of Springer Series in Computational Mathematics. Springer-Verlag, Berlin, 1996. MR1477665 (99a:65134)

[10] G. I. Shishkin. Grid approximation of singularly perturbed elliptic and parabolic equations (in Russian). Second Doctoral thesis. Keldysh Institute, Moscow, 1990.

[11] E. A. Volkov. On differential properties of solutions of boundary value problems for the Laplace and Poisson equations on a rectangle. Trudy Mat. Inst. Steklov, 77:89-112, 1965. MR0192077 (33:304)

Department of Mathematics, University of South Carolina, Columbia, South CarOLINA 29208

E-mail address: rbmjk@windstream.net

Institut für Numerische Mathematik, Technische Univeristät, Dresden, Germany

E-mail address: torsten.linss@tu-dresden.de

Department of Mathematics, National University of Ireland, Cork, Ireland

E-mail address: m.stynes@ucc.ie 